\title{
IS MILD COGNITIVE IMPAIRMENT A PRECURSOR OF ALZHEIMER'S DISEASE? SHORT REVIEW
}

\author{
Jana Janoutová', Omar Šerý ${ }^{2}$ Ladislav Hosák ${ }^{3}$, Vladimír Janout ${ }^{1}$ \\ 'Department of Epidemiology and Public Health, Faculty of Medicine, Ostrava University in Ostrava, Ostrava, Czech Republic \\ ${ }^{2}$ Institute of Animal Physiology and Genetics, Czech Academy of Sciences, Brno, Czech Republic \\ ${ }^{3}$ Department of Psychiatry, Charles University in Prague, School of Medicine in Hradec Králové, and University Hospital Hradec Králové, Hradec \\ Králové, Czech Republic
}

\begin{abstract}
SUMMARY
Mild Cognitive Impairment (MCI) may be a precursor of Alzheimer's disease (AD). There is a boundary area between normal aging and dementia. In practice, the term "age related cognitive decline" has been used interchangeably with "normal aging". Alternatively, the term "aging associated cognitive decline" was introduced and defined by a performance on a standardized cognitive scale focused on learning and memory, attention and cognitive speed, language, or visuoconstructional abilities. The term "mild cognitive impairment" was adopted by Petersen in 2004 to describe a period in the course of neurodegenerative disease where cognition is no longer normal relative to age expectations, however, daily functions are not sufficiently disrupted to correlate with the diagnosis of dementia. Most of the literature refers to the amnestic form of $\mathrm{MCl}$, which is likely a precursor of $A D$. The rate of conversion from amnestic form of $M C l$ to $A D$ is estimated to reach $10-15 \%$ per year. That is why $\mathrm{MCl}$ generated a great deal of research. When considering $\mathrm{MCl}$ a precursor of $A D$, it seems reasonable to study $A D$ genetic markers in the $M C l$ patients. In $A D$, association studies focus on genetic polymorphisms assumed to have an effect on the expression and modulation function of genes associated with AD pathogenesis (ApoE, APP, presenilin 1, presenilin 2, tau protein), and on polymorphisms related to metabolism of the aforementioned proteins (splicing, degradation). Neuropsychological assesment plays a substantial role in the diagnosis of $\mathrm{MCl}$, especially in the case of identification of different $\mathrm{MCl}$ subtypes or typical profiles of cognitive performance in prodromal phases of neurodegenerative diseases. The optimal composition of diet may increase an average age and prevent impairment of cognitive functions at the same time. Despite the progress in early diagnosis of $\mathrm{MCl}$ and dementia, further research is needed on differential diagnosis and treatment. In amnestic subtype of $\mathrm{MCl}$ some genetic markers may already be present, predicting possible future development of AD. Pointing to the need of secondary prevention, lifestyle modifications and possible early treatment could be implemented.
\end{abstract}

Key words: mild cognitive impairment, Alzheimer's dementia, terminology, genetics, neuropsychological testing, secondary prevention

Address for correspondence: V. Janout. Department of Epidemiology and Public Health, Faculty of Medicine, Ostrava University in Ostrava, Syllabova 19, 70300 Ostrava - Zábřeh, Czech Republic. E-mail: vladimir.janout@osu.cz

\section{What is the Mild Cognitive Impairment?}

There is a boundary area between normal aging and dementia. An extensive nomenclature exists to describe this boundary. Principally two forms of a cognitive change are associated with aging. One form involves benign changes in cognition associated with nonspecific histopathological brain changes. The other one is a malignant form which includes cognitive and behavioural changes with a specific brain histopathology.

In practice, the term "age related cognitive decline" has been used interchangeably with "normal aging" (1). On the other hand, there is the term "mild cognitive impairment" describing the course of a neurodegenerative disease where cognition is not normal relative to age expectations, but daily functions are not disturbed to qualify for the diagnosis of dementia (2).

Alternatively, the term "aging associated cognitive decline" (AACD) was introduced by Levy in 1994 (3) and defined by a performance on a standardized cognitive test that is at least one standard deviation below age-adjusted norms in at least one of any of the following cognitive domains: learning and memory, attention and cognitive speed, language, or visuoconstructional abilities. In addition to that, there should be no medical, psychiat- ric or neurological disorder that could cause cognitive impairment including dementia and normal activities of daily living should be preserved. Pantel et al. (4) demonstrated that parahippocampal brain volumes in patients meeting AACD criteria were intermediate between cognitively intact elderly individuals and individuals meeting criteria for Alzheimer's disease. Because of this, it seems suitable that AACD criteria could be a feasible approach for establishing diagnostic criteria for preclinical or potentially prodromal Alzheimer's dementia.

The term "mild cognitive impairment" (MCI) was initially introduced by Reisberg in 1982 but Petersen in 2004 (5) adopted this term to describe a period in the course of neurodegenerative disease where cognition is no longer normal relative to age expectations, but also where daily functions are not sufficiently disrupted to correlate with the diagnosis of dementia. In 2005, Petersen and Morris (6) distinguished amnestic and nonamnestic forms of MCI which might be associated with different underlying aetiologies like Alzheimer's disease or vascular dementia.

Most of the literature refers to the amnestic form of MCI, which is likely a precursor of Alzheimer's disease. The rate of conversion from amnestic form of MCI to Alzheimer's disease 
is estimated to be $10-15 \%$ per year. That is why MCI generated a great deal of research (7).

There are also some other emerging terms like "major neurocognitive disorder" and "minor neurocognitive disorder" but it is a question if they are going to be broadly adopted. There is a consensus in progress how to identify prodromes for nearly all neurodegenerative dementias and distinguish MCI from a typical cognitive aging.

We can start to use different biomarkers, neuroimaging, genetics and sensitive cognitive measures to identify dementia risk before cognitive impairment develops, but neuropsychological assessment will continue to play an important role in intervention planning, assessment of care needs and tracking the course of illness for the individual patient with dementia.

If we accept that MCI may be a precursor of Alzheimer's disease, it is useful to study genetic markers in MCI patients, which play a role in Alzheimer's disease. In the current genetic research, one of the most frequently used methods for studying multifactorial diseases such as $\mathrm{AD}$ are association studies. These are based on comparing allele and genotype frequencies between the studied groups of persons. In AD, association studies focus on genetic polymorphisms assumed to have an effect on the expression and modulation function of genes associated with $\mathrm{AD}$ pathogenesis (ApoE, APP, presenilin 1, presenilin 2, tau protein) and on polymorphisms related to metabolism of the aforementioned proteins (splicing, degradation). Selection of the so-called candidate genes results from the knowledge of pathological processes in $\mathrm{AD}$. In candidate genes, the so-called functional polymorphisms are most frequently selected, in which the effect on gene function has been proved (most often influencing the level of expression). As far the genetic risk factors are concerned, early-onset familial AD is assumed to be frequently caused by autosomal dominant mutations in the genes for APP (amyloid precursor protein), presenilin 1 and presenilin 2. This form of AD accounts for $2-5 \%$ of all AD cases. First-degree relatives of AD patients have a higher lifetime risk of developing the disease than the other populations. This increased risk and familial aggregation of AD cases are contributed to both genetic and other factors. From genetic factors, the risk may be partly due to the presence of the apolipoprotein E (APOE) e4 allele, but the other genes may also play a role in the development of the disease. The e 4 allele is so far the only confirmed genetic factor for the development of both early- and late-onset $\mathrm{AD}$. It is a factor increasing susceptibility to AD; however, it is neither necessary nor sufficient for its development. Homozygotes for the e 4 allele have a higher risk of AD and the age of its manifestation decreases. DNA polymorphisms of dozens of other candidate genes have been studied to reveal their relation to AD pathogenesis. Of those, some were shown as a risk. These are, for example, polymorphisms in the genes for nicastrin, ECE1, ACE, IDE, neprilysin, CHAT, ABCA1, CYP46, APOA1, IL-1, HLAA2, TNF A, cystatin C, PRNP, BACE1 and BACE2 (8).

In amnestic subtype of MCI some genetic markers may already be present, predicting possible future development of AD. In these persons, secondary prevention could be implemented in the form of both lifestyle modifications and possible early treatment.

Nikolaj et al. in their study stressed the fact that neuropsychological assesment plays a substantial role in the diagnosis of MCI, especially in the case of identification of different MCI subtypes or cognitive performance in prodromal phases of neurodegenera- tive diseases. Therefore, it is necessary to take into account the whole battery of presented methods such as neuropsychological assessment, magnetic resonance, biochemical, genetic and neuropsychiatric examination etc. (9).

One of the possibilities to increase average age in the population and prevent impairment of cognitive functions at the same time is optimal composition of a diet. Food usually does not supply organism with essential nutrients in adequate quantities. Out of different food components, it is supposed that PUFA, vitamins B9 and B12 or ginkgo biloba may contribute to optimal course of aging. Also individuals with mental disorders are consuming various dietary supplements with possible positive but also negative effects. It is therefore necessary to consult this topic with each patient individually (10).

Important biomarker of $\mathrm{AD}$ is early disturbance of episodic memory which may be present in MCI (11).

Another risk factor for dementia is smoking (12) which together with vascular changes caused by atherosclerosis may account for almost $40 \%$ of all dementia cases. Pathological processes subsequent to vascular changes caused by smoking bound together with the lack of oxygen or decreased blood flow then might cause the same effects increasing the probability of dementia onset even in a young person (13).

Even if a progress has been made in the early diagnosis of MCI and dementia, further research is needed to advance the utility of neuropsychological measures and genetic markers in differential diagnosis, prediction of progression and treatment planning $(14,15)$.

\section{Acknowledgement}

Supported by the Czech Ministry of Health grant project No. NT11152$6 / 2010$.

\section{REFERENCES}

1. Caccappolo-van Vliet E, Miozzo M, Marder K, Stern Y. Where do perseverations come from? Neurocase. 2003 Aug;9(4):297-307.

2. Mild cognitive impairment (MCI) [Internet]. Rochester (MN): Mayo Foundation for Medical Education and Research; 2012 [cited 2015 Nov 5]. Available from: http://www.mayoclinic.org/diseases-conditions/mildcognitive-impairment/basics/definition/con-20026392.

3. Levy R. Aging-associated cognitive decline. Working Party of the International Psychogeriatric Association in collaboration with the World Health Organization. Int Psychogeriatr. 1994;6(1):63-8. Erratum in: Int Psychogeriatr 1994;6(2):133.

4. Pantel J, Kratz B, Essig M, Schröder J. Parahippocampal volume deficits in subjects with aging-associated cognitive decline. Am J Psychiatry. 2003 Feb;160(2):379-82.

5. Petersen RC. Mild cognitive impairment as a diagnostic entity. J Intern Med. 2004 Sep;256(3):183-94.

6. Petersen RC, Morris JC. Mild cognitive impairment as a clinical entity and treatment target. Arch Neurol. 2005 Jul;62(7):1160-3; discussion 1167.

7. Sheardová K. Mild cognitive impairment in clinical practice. Psychiatr Praxi. 2010;11(2):62-5. (In Czech.)

8. Šré O, Povová J, Míšek I, Pešák L, Janout V. Molecular mechanisms of neuropathological changes in Alzheimer's disease: a review. Folia Neuropathol. 2013;51(1):1-9.

9. Nikolaj T, Bezdíčk O, Vyhnálek M, Hort J. Mild cognitive impairment: diagnostic unit or stadium preceding dementia? Českoslov Psychol. 2012;56(4):374-90. (In Czech.)

10. Raboch J. Cognitive functions, aging and dietary habits. Čes Slov Psychiatr. 2010;106(2):81-6. (In Czech.) 
11. Jirák R. Old and new diagnostic criteria for Alzheimer's disease in the conditions of the Czech Republic. Neurol Praxi. 2011;12(2):135-7. (In Czech.)

12. Merchant C, Tang MX, Albert S, Manly J, Stern Y, Mayeux R. Influence of smoking on the risk of Alzheimer's disease. Neurology. 1999 Apr 22;52(7):1408-12.

13. Peters R, Poulter R, Warner J, Beckett N, Burch L, Bulpitt C. Smoking, dementia and cognitive decline in the elderly, a systematic review. BMC Geriatr. 2008 Dec 23;8:36.
14. Smith GE, Bondi MW, editors. Mild cognitive impairment and dementia: definitions, diagnosis, and treatment. New York: Oxford University Press; 2013.

15. Clark LR, Schiehser DM, Weissberger GH, Salmon DP, Delis DC, Bondi MW. Specific measures of executive function predict cognitive decline in older adults. J Int Neuropsychol Soc. 2012 Jan;18(1):118-27.

Received April 29, 2015 Accepted in revised form December 7, 2015 